\title{
Traducción y validación del instrumento "Satisfacción de los padres con el cuidado pediátrico (SPCP)"
}

\author{
Rosalinda Garza Hernández, ${ }^{1, a, b}$ Concepción Meléndez Méndez, ${ }^{1, a, b}$ María del Socorro Rangel Torres, ${ }^{1, a, d}$ \\ Fernanda González Salinas, ${ }^{1, a, c}$ Hortensia Castañeda Hidalgo. ${ }^{1, c, e}$
}

Garza HR, Meléndez MC, Rangel TMS, González SF, Castañeda HH. Traducción y validación del instrumento "Satisfacción de los padres con el cuidado pediátrico (SPCP)". Cuid salud, jul-dic 2015; 2(2):195-202.

\section{RESUMEN}

Los instrumentos de recolección de datos válidos y confiables son un recurso elemental en la investigación en salud y cuando estos son generados en idiomas distintos se hace necesaria su traducción y validación. El objetivo del estudio fue traducir y validar el instrumento "Satisfacción de los padres con el cuidado pediátrico (SPCP)" en una población mexicana de padres de niños hospitalizados. Metodología: estudio cuantitativo de tipo descriptivo, se llevó a cabo en el Hospital No. 6 del IMSS de Cd. Madero, Tamaulipas. La muestra se calculó con un $95 \%$ de confianza y un $5 \%$ de margen de error, quedando conformada por 278 padres. Se utilizó un muestreo no probabilístico. El instrumento SPCP, consiste de 30 items. Para el análisis estadístico se realizó un análisis factorial, análisis de contenido y para conocer la confiabilidad del instrumento se hizo a través del coeficiente Alpha de Cronbach. Conclusiones: de acuerdo a los resultados obtenidos se puede considerar al instrumento SPCP válido y confiable, por lo tanto, los resultados de la versión española justifican su utilización para conocer el índice de satisfacción de los padres con el cuidado pediátrico en hospitalización.

Palabras clave: atención de enfermería, satisfacción del paciente, cuidado del niño.
Garza HR, Meléndez MC, Rangel TMS, González SF, Castañeda HH. Translation and validation of the questionnaire Parental satisfaction with pediatric care. Cuid salud, jul-dic 2015; 2(2):195-202.

\begin{abstract}
Valid and reliable instruments of data collection are an elementary resource in health research, when generated in different languages, their translation and validation is necessary. The purpose of the study was to translate and validate the instrument "Parents' Satisfaction with Pediatric Care (SPCP)" in a Mexican population of parents with hospitalized children. Methodology: Quantitative study of descriptive type. It was carried out in the Hospital No. 6 of the IMSS of Madero, Tamaulipas. The sample was calculated with $95 \%$ confidence and $5 \%$ margin of error, being formed by 78 parents. Non-probabilistic sampling was used. The SPCP instrument consists of 30 items. There were performed a factorial analysis, and content analysis for the statistical analysis, and to know the reliability of the instrument the Cronbach Alpha coefficient was applied. Conclusions: According to the results obtained, the SPCP instrument can be considered valid and reliable; therefore, the results of the Spanish version justify its use to know the rate of parents' satisfaction with pediatric care in hospitalization.
\end{abstract}

Keywords: Nursing care, patient satisfaction, child care.

\footnotetext{
1 Facultad de Enfermería de Tampico, Universidad Autónoma de Tamaulipas-México.

a Maestra en Ciencias de Enfermería.

b Profesora.

c Doctora.

d Directora.

Secretaría Académica.
} 


\section{INTRODUCCIÓN}

La satisfacción de los padres en una unidad de cuidado pediátrico es un tema vigente y de actualidad, que cobra más fuerza en tanto se puede medir y evaluar. Se debe tener en cuenta que lograr que el paciente tenga una buena percepción de la atención de salud que recibe en un centro hospitalario es tan importante como que se consiga la curación del padecimiento que presenta. Incluso, si esto último no se logra por cualquier motivo, si el paciente se siente bien atendido, es casi seguro que abandone la institución satisfecho. No así, cuando sucede lo contrario. En ese sentido, además de las naturales razones humanitarias, resulta de gran importancia para el sector de salud medir esta satisfacción y, derivado de los resultados de las encuestas que aplica, implementar medidas que conduzcan a una mejor atención de los usuarios.

Es importante considerar que un instrumento de evaluación que no tome en cuenta las experiencias previas y expectativas del paciente, será un instrumento incompleto para medir lo que se desea medir: la satisfacción del usuario. ${ }^{1}$ Pero en el caso de los pacientes pediátricos, hay que ir más allá y tomar en consideración lo que los padres han percibido en cuanto a la atención de salud otorgada a sus hijos. La evaluación en términos de satisfacción contiene un grupo complejo de variantes y de información útil para identificar dimensiones que afectan el bienestar individual y familiar para contrastar esa información con indicadores objetivos.

En una institución de salud, la intervención social al usuario involucra a éste y a su familia. $\mathrm{Si}$ se trata de un menor de edad (niño o adolescente) la fuente primaria de formación, información y convivencia será su núcleo de origen, por lo que evaluar la satisfacción de los padres con la atención de salud recibida por el paciente pediátrico debe ser un pilar fundamental para los proveedores de cuidado, en consecuencia, es oportuno contar con instrumentos de recolección de información confiables y válidos que ayuden a tener información adecuada que sustente la toma de decisiones. ${ }^{2}$

El concepto de cuidados centrados en la familia adquiere relevancia en niños, pues es necesario comprender las necesidades de los padres para facilitarles que sus experiencias sean lo más llevaderas posible. La satisfacción con los cuidados constituye el centro de un marco formado por respeto de sus necesidades, información adecuada, participación en la toma de decisiones, apoyo emocional y físico y, coordinación de los cuidados. ${ }^{3}$

La satisfacción del paciente es considerado un indicador confiable y válido de la calidad del cuidado, ya sea medido a través de la percepción del usuario o de los padres de la población pediátrica; ésta se define como un indicador de calidad que refleja la opinión en relación con dos aspectos prioritarios: el servicio de hospitalización y la atención recibida durante la estancia hospitalaria. Esta percepción se basa en la congruencia o acuerdo entre las expectativas del familiar del paciente pediátrico y la percepción de la experiencia. También puede considerarse que la satisfacción es una condición previa para el cuidado de calidad, donde se asume que una persona satisfecha cooperará más y demostrará una mayor conformidad a un tratamiento $\mathrm{y}$, por consiguiente, regresará a solicitar atención nuevamente. $^{4}$

Aunque existen limitaciones para precisar el concepto de satisfacción, pues representa una mezcla compleja de expectativas, necesidades y experiencias, ${ }^{5,6}$ puede definirse como una valoración positiva individual de diferentes aspectos de la atención de salud, en la que convergen variables psicológicas anteriores a la experiencia de recibir cuidados, como expectativas, valores, derechos, percepciones $\mathrm{y}$ comparaciones interpersonales. ${ }^{7}$

La satisfacción de los pacientes se extiende a sus familiares, ${ }^{8}$ principalmente en niños, donde el concepto de Cuidados Centrados en el Paciente se intercambia con el de Cuidados Centrados en la Familia. Éste implica una forma de entender los cuidados caracterizada por la estrecha interacción entre pacientes, familiares, médicos y enfermeras, jerarquiza aspectos emocionales, sociales, respeto por las características individuales del niño y su familia e información honesta para facilitar la toma de decisiones. ${ }^{9}$

En el caso de los pacientes que personalmente no pueden expresar sus puntos de vista, los miembros de la familia ayudan a determinar su satisfacción con el cuidado recibido. La calidad del cuidado puede ser evaluada a través de la satisfacción de los padres, basados en sus percepciones. ${ }^{10}$

Por todo lo expuesto este estudio tiene como objetivo traducir y validar un instrumento que mide la satisfacción de los padres con el cuidado 
pediátrico en hospitalización.

\section{MÉTODO}

\section{Tipo de estudio y población}

Estudio cuantitativo, exploratorio descriptivo, que se llevó a cabo en el Hospital No. 6 del Instituto Mexicano del Seguro Social de Madero, México; de enero a junio de 2014. La muestra se calculó con un $95 \%$ de confianza y un $5 \%$ de margen de error, quedando conformada por 278 padres de pacientes pediátricos hospitalizados. Se utilizó un muestreo no probabilístico tomando como criterios de inclusión: padres que habían tenido hospitalizado a su hijo en el área de pediatría por más de tres días y que deseaban por libre voluntad participar al momento de la recolección de la información. En el criterio de exclusión fueron considerados los padres de pacientes pediátricos en estado grave.

\section{Instrumento de recolección de datos}

El instrumento Satisfacción de los padres con el cuidado pediátrico-SPCP consiste en 30 ítems, con tres dimensiones del cuidado pediátrico: confort y servicio hospitalario, los cuidados de enfermería, los cuidados médicos. Lo concerniente a la dimensión de confort y servicio que brinda el hospital y determina la satisfacción de los padres es medida a través de 7 ítems (1-7) relacionados al procedimiento de admisión, limpieza y ergonomía del ambiente de la sala pediátrica; en relación a la satisfacción con los cuidados proporcionados por el personal de enfermería, es medida a través de 12 ítems (8-19) que involucran aspectos del cuidado tales como: atención, gentileza, acercamiento, información hacia el área médica, oportunidad en la administración de tratamientos y medicamentos, habilidad en el desarrollo de procedimientos, información hacia los padres de manera clara e inclusión en la toma de decisiones de los mismos en el cuidado pediátrico. La dimensión del cuidado médico es analizada a través de 11 ítems (20-30), que indaga aspectos tales como: conocimiento de los mismos de la historia clínica, son cuidadosos y considerados, muestran habilidades, conocimiento, disponibilidad al solicitárseles, respondieron ante las condiciones del paciente pediátrico, brindaron información clara, explícita y honesta y disipación de dudas a los padres e inclusión en la toma de decisiones del cuidado pediátrico.

Las respuestas se miden mediante escala de tipo Likert con 5 tipos de respuesta de 1 a 5 , en donde esta cinco corresponde a muy satisfecho, 4 a satisfecho, 3 a moderadamente satisfecho, 2 a poco satisfecho y 1 a insatisfecho. Para determinar el nivel de satisfacción con el cuidado pediátrico los resultados fueron transformados a índices de cero a 100 , en donde 150 corresponde al valor máximo y 30 el valor mínimo, siendo que arriba de 60 se considera como satisfecho y arriba de 80 se consideraran como muy satisfecho. Todos los ítems fueron redactados de manera positiva lo que significa que a mayor puntuación mayor satisfacción.

Sobre la traducción del instrumento: primero se llevó a cabo la traducción-retrotraducción del instrumento que en su versión en inglés es el "Pediatric Family Satisfaction Questionnaire" elaborado por Budreau y Chase $^{11}$ y rediseñado por Bragadottir y Reed. $^{12}$ La traducciónretrotraducción fue realizada por dos expertas en investigación y bilingües. Una vez que se consideró que la traducción era correcta y que no hubo cambios en la retrotraducción, se aplicó el instrumento a seis personas de habla castellana, expertas en el cuidado de pacientes pediátricos para validar el instrumento traducido, considerando en forma subjetiva si éste en general $y$ en cada uno de sus dominios evaluaban adecuadamente la satisfacción de los padres con los cuidados recibidos por el hijo durante la hospitalización. Cada una de las expertas realizó su trabajo en forma individual y sin estar al tanto de las respuestas de las demás. Los resultados de esta etapa indicaron que no había necesidad de modificar ninguna palabra.

\section{Procedimiento de recolección de datos}

Para la recolección de la información se utilizó el instrumento traducido "Satisfacción de los padres con el cuidado pediátrico (SPCP)" y una cédula de datos sociodemográficos, para conocer información relacionada al parentesco del familiar cuidador, escolaridad y edad. Se aplicó el instrumento a los 278 padres de la muestra.

Los padres del estudio se identificaron conforme a los servicios del hospital, una vez identificados, se les invitó a participar en el estudio, explicándoles que su participación consistiría en contestar un cuestionario escrito, se les explicó que este tipo de recolección de datos es importante para tener instrumentos de investigación que ayuden a conocer el nivel de satisfacción con el cuidado pediátrico otorgado a su hijo durante la hospitalización, además se les notificó que dicha información era confidencial para uso exclusivo de la investigación, una vez que 
aceptaron participar en el estudio se solicitó por escrito la firma del consentimiento informado.

\section{Análisis de datos}

Para la captura de información se utilizó el programa estadístico SPSS (Statiscal Package for the Social Sciences, versión 19), para describir sociodemográficamente a la muestra, se hizo mediante la estadística descriptiva y para el procedimiento de la validación se realizó una estimación de las comunalidades y una vez obtenidas estas, se procedió a llevar a cabo una prueba de rotación tipo varimax, posteriormente cuando se obtuvo la estructura factorial, se agruparon las variables según su carga factorial. Por último para identificar la confiabilidad interna del instrumento, se llevó a cabo una prueba de Alpha de Cronbach.

\section{Consideraciones éticas}

Este estudio se realizó conforme a lo dispuesto en el Reglamento de la Ley General de
Salud en materia de investigación, de la Secretaría de Salud en México ${ }^{13}$ y se sometió a revisión al Comité de Ética en Investigación de la Facultad de Enfermería de Tampico de la Universidad Autónoma de Tamaulipas (Oficio No 16/2014), así como de la institución participante.

\section{RESULTADOS}

En el estudio participaron 278 padres que tenían un hijo hospitalizado en el servicio de pediatría, de los cuales el $64 \%$ era mujer y $36 \%$ hombre; el $8 \%$ no contaba con educación escolarizada, el 53\% tenía una educación básica (primaria y/o secundaria), 25\% contaba con educación media superior y $14 \%$ tenía preparación profesional o de posgrado.

Se realizó una estimación de las comunalidades de las variables (ítems), mediante el método de extracción denominado componentes principales con rotación de la matriz resultante tipo varimax (tabla 1).

Tabla 1. Estimación de las comunalidades.

\begin{tabular}{|c|c|c|}
\hline Comunalidades & Inicial & Extracción \\
\hline En el servicio hospitalario El procedimiento de admisión fue sencillo & 1,000 & ,331 \\
\hline En el confort hospitalario El cuarto y la unidad del paciente estaban limpios & 1,000 & , 479 \\
\hline En el confort hospitalario La decoración y amueblado era adecuado & 1,000 & , 455 \\
\hline En el confort hospitalario El paciente no fue molestado por ruidos & 1,000 & ,534 \\
\hline En el servicio hospitalario El personal del hospital trabajó como un equipo & 1,000 & ,571 \\
\hline En el servicio hospitalario Fuimos preparados adecuadamente para el alta del paciente & 1,000 & 615 \\
\hline En general estamos satisfechos con el cuidado recibido en el servicio & 1,000 & ,585 \\
\hline Las enfermeras fueron consideradas y cuidadosas & 1,000 & ,543 \\
\hline Las enfermeras fueron amables con el paciente & 1,000 & ,554 \\
\hline Las enfermeras revisaron la condición del paciente cuidadosamente & 1,000 & 686 \\
\hline Las enfermeras notificaban al médico cuando era necesario & 1,000 & ,697 \\
\hline Las enfermeras estuvieron al pendiente de los cambios durante el tratamiento & 1,000 & ,708 \\
\hline Las enfermeras dieron el tratamiento y los medicamentos a tiempo & 1,000 & ,533 \\
\hline Las enfermeras fueron hábiles con los procedimientos y el equipo & 1,000 & ,682 \\
\hline Las enfermeras nos mantenían informados & 1,000 & 619 \\
\hline Las enfermeras nos respondieron nuestras preguntas claramente & 1,000 & ,554 \\
\hline $\begin{array}{l}\text { Las enfermeras explicaron la condición del paciente y su cuidado, en términos que nosotros } \\
\text { entendiéramos }\end{array}$ & 1,000 & ,546 \\
\hline Las enfermeras escuchaban lo que teníamos que decir & 1,000 & 649 \\
\hline Las enfermera Nos incluyeron al tomar decisiones y al planear el cuidado de mi hijo (a) & 1,000 & ,721 \\
\hline Los médicos Fueron considerados y cuidadosos & 1,000 & ,635 \\
\hline Los médicos estaban familiarizados con la historia médica del paciente & 1,000 & 635 \\
\hline Los médicos tenían conocimientos y habilidades & 1,000 & ,617 \\
\hline Los médicos estaban disponibles cuando se les llamaba o eran necesitados & 1,000 &, 527 \\
\hline Los medico respondían con prontitud a los cambios en la condición del paciente & 1,000 & ,705 \\
\hline Los médicos tuvieron una comunicación clara y honesta con nosotros & 1,000 & ,705 \\
\hline Los médicos nos dieron información acerca de los tratamientos y exámenes antes de hacerlos & 1,000 & ,755 \\
\hline $\begin{array}{l}\text { Los médicos nos mantuvieron informados de los resultados de las pruebas y de los cambios en } \\
\text { la condición del paciente }\end{array}$ & 1,000 & ,802 \\
\hline Los médicos nos dieron explicaciones completas & 1,000 & ,782 \\
\hline Los médicos respondieron nuestras preguntas claramente & 1,000 & ,782 \\
\hline Los médicos nos incluyeron al tomar decisiones y a planear el cuidado de mi hijo(a) & 1,000 & ,810 \\
\hline
\end{tabular}


A continuación se obtuvo la matriz de la estructura factorial en donde se agrupan los ítems en cada uno de los tres componentes. Las variables fueron agrupadas en los componentes de acuerdo al siguiente criterio: cargas factoriales mayores o superiores a 0,3; en el caso de las variables que saturaron en dos o más componentes, estas quedaron agrupadas en el de mayor carga factorial y que correspondiera conceptualmente al componente (tabla 2).

Tabla 2. Matriz de componentes rotados.

\begin{tabular}{|c|c|c|c|}
\hline \multirow{2}{*}{ Ítems } & \multicolumn{3}{|c|}{ Componente } \\
\hline & $\mathrm{C} 1$ & $\mathrm{C} 2$ & $\mathrm{C} 3$ \\
\hline En el servicio hospitalario el procedimiento de admisión fue sencillo & & & ,544 \\
\hline En el confort hospitalario el cuarto y la unidad del paciente estaban limpios & & & ,667 \\
\hline En el confort hospitalario la decoración y amueblado era adecuado & & &, 651 \\
\hline En el confort hospitalario el paciente no fue molestado por ruidos & & & ,723 \\
\hline En el servicio hospitalario el personal del hospital trabajo como un equipo & & & ,675 \\
\hline En el servicio hospitalario fuimos preparados adecuadamente para el alta del paciente & & & ,758 \\
\hline En general estamos satisfechos con el cuidado recibido en el servicio & & & ,669 \\
\hline Las enfermeras fueron consideradas y cuidadosas & & ,670 & \\
\hline Las enfermeras fueron amables con el paciente & & ,670 & \\
\hline Las enfermeras revisaron la condición del paciente cuidadosamente & & ,785 & \\
\hline Las enfermeras notificaban al médico cuando era necesario & & ,795 & \\
\hline Las enfermeras estuvieron al pendiente de los cambios durante el tratamiento & & ,800 & \\
\hline Las enfermeras dieron el tratamiento y los medicamentos a tiempo & & ,627 & \\
\hline Las enfermeras fueron hábiles con los procedimientos y el equipo & & ,745 & \\
\hline Las enfermeras nos mantenían informados & & ,657 & \\
\hline Las enfermeras nos respondieron nuestras preguntas claramente & & ,611 & \\
\hline $\begin{array}{l}\text { Las enfermeras explicaron la condición del paciente y su cuidado, en términos que nosotros } \\
\text { entendiéramos }\end{array}$ & & ,607 & \\
\hline Las enfermeras escuchaban lo que teníamos que decir & & ,696 & \\
\hline Las enfermera nos incluyeron al tomar decisiones y al planear el cuidado de mi hijo (a) & & ,730 & \\
\hline Los médicos fueron considerados y cuidadosos & ,703 & & \\
\hline Los médicos estaban familiarizados con la historia médica del paciente & ,730 & & \\
\hline Los médicos tenían conocimientos y habilidades & ,736 & & \\
\hline Los médicos estaban disponibles cuando se les llamaban o eran necesitados & 692 & & \\
\hline Los medico respondían con prontitud a los cambios en la condición del paciente & ,780 & & \\
\hline Los médicos tuvieron una comunicación clara y honesta con nosotros & ,789 & & \\
\hline Los médicos nos dieron información acerca de los tratamientos y exámenes antes de hacerlos & ,798 & & \\
\hline $\begin{array}{l}\text { Los médicos nos mantuvieron informados de los resultados de las pruebas y de los cambios en } \\
\text { la condición del paciente }\end{array}$ & ,839 & & \\
\hline Los médicos nos dieron explicaciones completas & ,843 & & \\
\hline Los médicos respondieron nuestras preguntas claramente & ,847 & & \\
\hline Los médicos nos incluyeron al tomar decisiones y a planear el cuidado de mi hijo(a) &, 850 & & \\
\hline
\end{tabular}

Los componentes fueron conceptualizados de la manera siguiente: cuidados médicos (C1), cuidados de enfermería (C2) y confort y servicio hospitalario (C3). Después de analizar a través del juicio de expertos la validez de los ítems el instrumento quedó de la siguiente manera (tabla 3). 
Tabla 3. Especificaciones del cuestionario: Satisfacción de los padres con el cuidado pediátrico en hospitalización-SPCP.

\begin{tabular}{|c|c|c|}
\hline Componentes & Definición & Indicadores \\
\hline $\begin{array}{l}\text { C1 } \\
\text { Cuidados } \\
\text { médicos }\end{array}$ & $\begin{array}{l}\text { Indaga sobre: conocimiento de la } \\
\text { historia clínica, son cuidadosos, } \\
\text { hábiles y considerados, } \\
\text { disponibilidad, respondieron ante } \\
\text { las condiciones del paciente } \\
\text { pediátrico, brindaron información } \\
\text { clara, explícita y honesta, } \\
\text { disipación de dudas a los padres, } \\
\text { e inclusión en la toma de } \\
\text { decisiones del cuidado pediátrico. }\end{array}$ & $\begin{array}{l}\text { Los médicos Fueron considerados y cuidadosos } \\
\text { Los médicos estaban familiarizados con la historia médica } \\
\text { del paciente } \\
\text { Los médicos tenían conocimientos y habilidades } \\
\text { Los médicos estaban disponibles cuando se les llamaban o } \\
\text { eran necesitados } \\
\text { Los medico respondían con prontitud a los cambios en la } \\
\text { condición del paciente } \\
\text { Los médicos tuvieron una comunicación clara y honesta con } \\
\text { nosotros } \\
\text { Los médicos nos dieron información acerca de los } \\
\text { tratamientos y exámenes antes de hacerlos } \\
\text { Los médicos nos mantuvieron informados de los resultados } \\
\text { de las pruebas y de los cambios en la condición del paciente } \\
\text { Los médicos nos dieron explicaciones completas } \\
\text { Los médicos respondieron nuestras preguntas claramente } \\
\text { Los médicos nos incluyeron al tomar decisiones y a planear } \\
\text { el cuidado de mi hijo(a) }\end{array}$ \\
\hline $\begin{array}{l}\text { C2 } \\
\text { Cuidados de } \\
\text { enfermería }\end{array}$ & $\begin{array}{l}\text { Involucra aspectos del cuidado } \\
\text { como: atención, gentileza, } \\
\text { acercamiento, oportunidad en la } \\
\text { administración de tratamientos y } \\
\text { medicamentos, habilidad en el } \\
\text { desarrollo de procedimientos, } \\
\text { información hacia los padres de } \\
\text { manera clara e inclusión en la } \\
\text { toma de decisiones de los mismos } \\
\text { en el cuidado pediátrico. }\end{array}$ & $\begin{array}{l}\text { Las enfermeras fueron consideradas y cuidadosas } \\
\text { Las enfermeras fueron amables con el paciente } \\
\text { Las enfermeras revisaron la condición del paciente } \\
\text { cuidadosamente } \\
\text { Las enfermeras notificaban al médico cuando era necesario } \\
\text { Las enfermeras estuvieron al pendiente de los cambios } \\
\text { durante el tratamiento } \\
\text { Las enfermeras dieron el tratamiento y los medicamentos a } \\
\text { tiempo } \\
\text { Las enfermeras fueron hábiles con los procedimientos y el } \\
\text { equipo } \\
\text { Las enfermeras nos mantenían informados } \\
\text { Las enfermeras nos respondieron nuestras preguntas } \\
\text { claramente } \\
\text { Las enfermeras explicaron la condición del paciente y su } \\
\text { cuidado, en términos que nosotros entendiéramos } \\
\text { Las enfermeras escuchaban lo que teníamos que decir } \\
\text { Las enfermera Nos incluyeron al tomar decisiones y al } \\
\text { planear el cuidado de mi hijo (a) }\end{array}$ \\
\hline $\begin{array}{c}\text { C3 } \\
\text { Confort y } \\
\text { servicio } \\
\text { hospitalario }\end{array}$ & $\begin{array}{l}\text { Relacionado al procedimiento de } \\
\text { admisión, limpieza y ergonomía } \\
\text { del ambiente de la sala pediátrica }\end{array}$ & $\begin{array}{l}\text { En el servicio hospitalario El procedimiento de admisión } \\
\text { fue sencillo } \\
\text { En el confort hospitalario El cuarto y la unidad del paciente } \\
\text { estaban limpios } \\
\text { En el confort hospitalario La decoración y amueblado era } \\
\text { adecuado } \\
\text { En el confort hospitalario El paciente no fue molestado por } \\
\text { ruidos } \\
\text { En el servicio hospitalario El personal del hospital trabajo } \\
\text { como un equipo } \\
\text { En el servicio hospitalario Fuimos preparados } \\
\text { adecuadamente para el alta del paciente } \\
\text { En general estamos satisfechos con el cuidado recibido en el } \\
\text { servicio }\end{array}$ \\
\hline
\end{tabular}

Se utilizó el Alpha de Cronbrach debido a su elevada sensibilidad para detectar fuentes de error de medición atribuibles al muestreo de factores situacionales así como de contenido de los reactivos. La tabla 4 presenta los índices de confiabilidad correspondientes a cada uno de los 
tres componentes, todos ellos arriba de 0,80 lo cuál indica que son índices altamente aceptables.

Tabla 4. Índice de confiabilidad por componentes

\begin{tabular}{cccc}
\hline Componente & Media & Varianza & $\begin{array}{c}\text { Alfa de } \\
\text { Cronbach }\end{array}$ \\
\hline C1 & 4,49 & 0,004 & 0,96 \\
C2 & 4,54 & 0,001 & 0,94 \\
C3 & 4,26 & 0,019 & 0,82 \\
\hline
\end{tabular}

\section{DISCUSIÓN}

Se puede decir que: La relevancia de este estudio está dada por la oportunidad de contar con un instrumento simple y de alta validez clínica concurrente y predictiva que permite valorar adecuadamente la satisfacción de los padres con el cuidado pediátrico. La consistencia interna de cada uno de los factores encontrados es considerablemente alta $(0,82-0,96)$, lo que demuestra que las preguntas planteadas para cada factor realmente corresponden a dicho factor $\mathrm{y}$ confirman la multidimensionalidad del constructo. En este estudio se observa que la satisfacción no es un constructo unidimensional debido al número de factores encontrados: cuidados médicos (C1), cuidados de enfermería (C2) y confort y servicio hospitalario (C3). Para este análisis se consideraron como ítems aceptables aquéllos que presentaron comunalidades superiores a 0,3 , en total fueron 30 los ítems que cumplieron esta condición (tabla 1).

Los ítems 30, 27, 28 y 29 fueron los mejores explicados por el modelo factorial obtenido, ya que sus porcentajes de varianza son de $81 \%, 80,2 \%$, 78,2 y 78,2\% respectivamente; los ítems 1,3 y 2 resultaron los menos explicados pues el modelo factorial solo puede reproducir el $33,1 \%, 45,5 \%$ y $47,9 \%$ respectivamente de sus variabilidades originales (tabla 1$)$.

Algunas limitaciones pueden ser consideradas en el estudio, sobre todo considerando la población participante, dado que en los criterios de inclusión no se obtuvieron datos de padres de pacientes, por ejemplo, con hijos fallecidos o egresados con secuelas o a continuar con atención domiciliaria. Aunque estos criterios de exclusión se emplean en estudios similares, sería interesante conocer otras opiniones. Sería oportuno aplicar el instrumento validado para identificar la Satisfacción de los padres con el cuidado pediátrico en contexto mexicano y latinoamericano para seguir analizando su utilidad. También será interesante profundizar en este tema inclusive con nuevas indagaciones de tipo cualitativo.

\section{CONCLUSIONES}

La validación del instrumento resultó adecuada en la población estudiada. Es importante que se realice unificación de criterios y se utilicen instrumentos válidos y reproducibles a fin de lograr una estandarización de la evaluación de la satisfacción y la comparabilidad de los resultados obtenidos entre las diferentes instituciones $o$ servicios.

Es posible evaluar la satisfacción de los padres con los cuidados recibidos por sus hijos durante la hospitalización. El instrumento empleado es adecuado para esta población.

La utilización indiscriminada y variada de instrumentos para medir satisfacción sin que hayan sido sometidos a pruebas de validez $y$ confiabilidad, podrían no reflejar la realidad de la satisfacción del usuario o sus familiares y dificultar la comparabilidad; lo cual se puede ver traducido en la toma de decisiones equivocadas generadas a partir de la evaluación de los servicios.

Finalmente, existe la necesidad de aplicación de instrumentos válidos y reproducibles que evalúen satisfacción del usuario en los diferentes servicios y que permitan la comparabilidad, teniendo en cuenta las características propias del sistema de salud y de la población.

\section{Declaración de financiamiento y de conflictos de interés:}

El estudio fue financiado por los autores; declaran no tener conflictos de interés.

\section{Contribución de autoría:}

RGH, CMM, MSRT, FGS, HCH: Concepción y diseño del estudio, recolección de los datos, análisis e interpretación de los resultados y aprobación de la versión que será publicada.

\section{Correspondencia:}

Hortensia Castañeda-Hidalgo

Correo electrónico: hcastane@docentes.uat.edu.mx 


\section{REFERENCIAS BIBLIOGRÁFICAS}

1. Hamui SL, Fuentes GR, Aguirre FR, Ramírez de la Roche OF. Expectativas y experiencias de los usuarios del Sistema de Salud en México. Un estudio de la satisfacción con la atención médica [internet] Distrito Federal: Universidad Nacional Autónoma de México; 2013 [citado 5 de ago 2015]. 208 p. Disponible en: http://www.facmed.unam.mx/bmnd/Satisfaccio nAtencionMedica.pdf

2. Obando HIM. El niño enfermo y su familia. Revista de trabajo social [internet]. 1993 [citado 21 de jul 2014]; 18(40): 363-376. Disponible

en: http://www.binasss.sa.cr/revistas/ts/v18n40199 3/art03.pdf

3. Latour JM, Van Goudoever JB, Hazelzet JA. Parent satisfaction in the Pediatric ICU. Pediatr Clin North Am [serie de internet]. Jun 2008 [citado 17 de ago 2015]; 55(3): 779.790. Disponible en: https://www.ncbi.nlm.nih.gov/pubmed/185017 $\underline{65}$

4. Serrano-del Rosal R, Lorríente-Arín N. La anatomía de la satisfacción del paciente. Salud Pública Mex [serie de internet]. Mar-abr 2008 [citado 14 de dic 2014]; 50(2): 162172. Disponible en: http://www.scielosp.org/pdf/spm/v50n2/10.pdf

5. Silva A. A framework for measuring responsiveness. GPE. Discussion Paper Series $\mathrm{N}^{\circ} 32$ [internet]. s.n.: Irganización Mundial de la Salud; s.f. [citado 22 de ago 2015]; 42p. Disponible en: http://www.who.int/healthinfo/paper32.pdf

6. Thompson AG, Suñol R. Expectations as determinants of patients satisfaction: concepts, theory and evidence. Int J Qual Health Care [serie de internet]. Jun 1995. [citado 19 de ago 2015]; 7(2):127-141. Disponible en: http://citeseerx.ist.psu.edu/viewdoc/download? $\underline{\text { doi }=10.1 \cdot 1.1014 .88 \& \mathrm{rep}=\mathrm{rep} 1 \& \text { type }=\mathrm{pdf}}$

7. Linder-Pelz SU. Toward a theory of patient satisfaction. Soc Sci Med [serie de internet]. Feb 1982. [citado 13 de mar 2015]; 16(5):577-82: Disponible en: https://www.ncbi.nlm.nih.gov/pubmed/710099 $\underline{0}$
8. Fornaris FM, Castillo PM, Rodríguez MA, Arias AZ, Acosta PY. Evaluación de la satisfacción de los usuarios con la atención estomatológica al menor de 19 años. Medisan [serie de internet]. 2006. [citado 21 de ago 2015];10 (2): [aproximadamente 06 aludas]. Disponible en: http://bvs.sld.cu/revistas/san/vol10_2_06/san05 206.pdf

9. Colunga RC, López MM, Aguayo AG, Canales MJ. Calidad de atención y satisfacción del usuario con cita previa en medicina familiar de Guadalajara. Rev Cubana de Salud Pública [serie de internet]. 2007 [citado 6 de ago 2015]; 33 (3): [aproximadamente 05 laudas]. Disponible en: http://bvs.sld.cu/revistas/spu/vol33_3_07/spu12 $\underline{307 . h t m}$

10. Roberti SM, Fitzpatric JJ. Assessing family satisfaction with care of critically ill patients: a pilot study. Critical Care Nurse [serie de internet]. Dec 2010 [citado 8 de ago 2015]; 30(6): 18-26. Disponible en: http://ccn.aacnjournals.org/content/30/6/18.full. pdf + html

11. Budreau G, Chase L. A family-centered approach to the development of a pediatric family satisfaction questionnaire. Pediatric Nursing [serie de internet].Nov-dec 1994. [citado 11 de ago 2014]; 20(6): 604-608. Disponible en: https://www.ncbi.nlm.nih.gov/pubmed/770846 $\underline{3}$

12. Bragadóttir H, Reed D. Psychometric Instrument Evaluation: The Pediatric Family Satisfaction Questionnaire. Pediatr Nurs [serie de internet]. Sep-Oct 2002. [citado 17 de ago 2015]; 28 (5): 475-82. Disponible en: https://www.ncbi.nlm.nih.gov/labs/articles/124 $\underline{24983 /}$

13. Secretaría de Salud. Reglamento de la ley general de salud en materia de Investigación para la salud [internet]. México DF: Secretaria de salud; 2014 [citado 14 de ago 2015]. 09p. Disponible en: https://archivos.juridicas.unam.mx/www/bjv/li bros/5/2292/63.pdf 